THE EXTRAGALACTIC ISM 


\title{
STAR FORMATION IN GALACTIC NUCLEI
}

\author{
Roberto Terlevich \\ Royal Greenwich Observatory, \\ Herstmonceux, BN27 1RP, \\ U.K.
}

Summary. The term AGN or Active Galactic Nucleus, although with rather illdefined boundaries, signifies extragalactic systems with non-stellar nuclear activity. Nonstellar activity indicators are: high luminosity, presence of broad emission lines, variability, nuclear UV excess, $x$-ray emission, radio emission, variable polarization, jets, etc.

The list of AGNs includes LINERs, Seyfert galaxies, QSOs (optically selected), Quasars (radio selected), narrow-line and broad-line radio galaxies, optically violent variable Quasars and BL Lac type objects. The large luminosity of AGNs, is believed to derive from gravitational energy and is usually associated with an accretion disk circling a super-massive blackhole. It is also customary to assume that the size of the emitting region is limited by its variability to a few light weeks and that the very broad emission lines (FWHI 5000 $\mathrm{km} \mathrm{s}^{-1}$ ) of the so called Broad Line Region (BLR) are consistent with photoionization of high density filaments orbiting the inner few parsecs of the AGN.

Here I will discuss the possibility that the energy source in at least some AGNs is a burst of star formation. In this scenario the BLR is the product of SN remnants evolving in high density medium. I will show that the observed variability of the broad line region in Seyferts type 1 and QSOs is consistent with the predictions of the starburst scenario, and that the total luminosity of even the most luminous QSOs is well inside the expectations of dissipative galaxy formation scenarios.

I. Overview of the scenario. There is a fundamental dichotomy, first reported by Heckmann et al. (1980), in the distribution of Hubble types of galaxies with emission line nuclei. While most galaxies with active nuclei have Hubble types earlier than Sbc, those with HII region type nuclei have Hubble types later than Sbc.

Independently of the origin of the bi-modal Hubble type distribution, (see Terlevich et al. 1987) the fact remains that is only in luminous bulges that metal rich stellar populations are found (Cowley et al. 1982), and when those bulges show nuclear emission lines, they are systematically classified as 'active' as opposed to HII region type.

For the past few years a large part of my work has been devoted to the study of regions with active star formation. In particular the study of the intrinsic properties of giant extragalactic HII regions like 30-Doradus in the LMC and HII galaxies like IIZw40 and IZw18. HII galaxies have small dimensions, spheroidal shape, a young (very hot) stellar population and spectroscopic properties indistinguishable from those of giant extragalactic HII regions. With Jorge Melnick, Mariano Moles and Josefa Masegosa, I finished a large scale 
spectrophotometric survey of about 500 HII galaxies selected from objective prism Schmidt surveys. A totally unexpected result from that study was the relatively narrow metallicity distribution of galaxies (FWHM 0.7 Dex). We found only few galaxies showing oxygen abundance less than 0.1 solar and no galaxy with oxygen abundance larger than 1.5 times solar (Terlevich 1988).

Where are then the luminous metal rich bursts of star formation? With Jorge Melnick, Mariano Moles, Angeles Díaz and Elena Terlevich I started to estimate the properties of bursts of star formation at high metallicities. We found that unlike the low metallicity case, metal rich massive stars have their evolution fundamentally affected by mass-loss in the form of stellar winds. Without exception all evolutionary computations find the same differences between conservative, $\dot{M}=0$, and mass-losing models found initially by Tanaka (1966); namely a change in the $\mathrm{H}$ and $\mathrm{He}$ burning scale and an evolution towards high temperatures after hydrogen exhaustion. During the blueward evolution the star reaches effective temperatures well in excess of those typical of the ZAMS. Wolf-Rayet stars with massive progenitors $\left(\mathrm{M}>60 \mathrm{M}_{\odot}\right)$ are believed to be in the blueward evolutionary stage (Conti 1976, Chiosi 1981, Maeder 1983). The Wolf-Rayet stage is reached after a highly unstable phase, corresponding to the minimum effective temperature ever reached by the star $(\sim 15,000 \mathrm{~K})$ and roughly before the onset of helium burning. Luminous blue variables, like the Hubble-Sandage variables, $\eta$ Carina and P-Cygni type stars are believed to be in this unstable phase. The most recent evolutionary star models for solar composition and incorporating mass loss and overshooting, indicates that by the end of the helium burning phase the effective temperature reaches up to $200,000 \mathrm{~K}$ and their bolometric luminosity could be up to a factor of 2 larger than at the ZAMS. We call these extremely hot and luminous Wolf-Rayet stars WARMERS.

WARMERS do exist. Barlow and Cohen (1982) have indicated the existence of a new class of extremely hot Wolf-Rayet stars (WO stars), characterized by the presence of high ionization emission lines of oxygen and carbon (OIV, CIV) in their optical and UV spectra. Davidson and Kinman (1982) reported observations of an HII region in the dwarf galaxy IC1613 ionized by a Wolf-Rayet star with broad OVI and CIV in emission probably an extreme WC5 or even WC4. Using the spectrum of the HII region they determine that most of the luminosity is emitted at an energy of $37 \mathrm{eV}$ similar to the peak of a $110,000 \mathrm{~K}$ blackbody spectrum. The estimated bolometric luminosity is $1.3 \times 10^{5} \mathrm{~L}_{\odot}$.

With Jorge Melnick I investigated the changes that a population of WARMERS will introduce in the emitted spectrum of a young metal rich cluster (Terlevich and Melnick 1985; hereafter TM85). Using Maeder's isochrones, we studied the evolution of a burst of star formation using our computer code IMF. This program computes from the theoretical isochrones at stellar atmospheres the integrated spectrum of a star cluster as a function of age; then calculates the emission line spectrum of the associated HII region using the photoionization code CLOUDY developed by Gary Ferland.

We found that the emitted spectrum of a metal rich HII region suffers a qualitative change after about $3 \mathrm{Myr}$ of evolution, when the most massive stars reach the WARMER 
phase. In a very short time the ionizing spectrum of the cluster is fundamentally modified by the appearance of a luminous and hot component, the WARMER component. Consequently the emission line spectrum is transformed from that of a typical low excitation HII region into a high excitation Seyfert type 2. Following the evolution still further, shows that after $5 \mathrm{Myr}$ as the ionizing flux decreases and therefore the ionization parameter also decreases, the Seyfert type 2 nucleus becomes a Liner.

In brief, TM85 have shown that the 'traditional' method of using the Baldwin, Phillips and Terlevich (1981) diagnostic diagrams to classify emission line regions should be used with care. Power law type ionizing continuum can be the result of a few WARMERs modifying the emitted UV spectrum of a young cluster. The best evolutionary star models predict this behaviour for solar and over solar abundances.

This result raised the possibility of a direct relation between star formation and some forms of nuclear activity. This possibility has been discussed in the literature by several authors (Pronik 1973, Adams and Weedman 1975, Harwit and Pacini 1975, Osterbrock 1978, Weedman 1983, Perry and Dyson 1985). Some researchers have preferred the view that after the star formation event, the resulting cluster of compact remnants will eventually coalesce and form a massive blackhole (Rees 1978, 1984), while others suggest that the gas lost during the evolution of the massive stars is able to fall into the gravitational potential of the bulge, cool and form a blackhole (Norman and Scoville 1988). All these scenarios have a common point, they have to start the evolution with extremely compact clusters with large velocity dispersions to allow the formation of a blackhole.

Another possibility originally suggested by Shklovskii (1960), and further developed by Field (1964) and McCrea (1976) and more recently by Terlevich et al. (1987), Terlevich and Melnick (1987) and Terlevich (1989) is that no blackhole forms and that all the observed properties of at least some AGNs are the direct consequence of a nuclear starburst.

The observed variability of the Broad Line Region (BLR) in Seyferts type 1 and QSOs, the line profiles and line ratios, total luminosity and small size of the BLR, poses difficulties for the starburst scenario. In this paper I will discuss these points.

II. The BLR in the starburst scenario. With Melnick and Moles (1987; hereafter TMM87), I extended the work of TM85 to include the supernova phase in the evolution of the nuclear starburst. In that paper we postulated that the observed properties of Seyfert type 1 nuclei may also be related to nuclear starburst activity. We distinguished two different supernova activity phases,

1 - a SN type Ib at the end of the life-time of the most massive stars ( $\left.M>25 M_{\odot}\right)$. These SNe have Wolf-Rayet/WARMERS progenitors and they are predicted to be optically dim and radio loud. This stage is associated with the Seyfert 2 phase.

2 - a SN type II phase at the end of the life-time of intermediate mass stars $(8<$ $\left.M<25 \mathrm{M}_{\odot}\right)$. These SNe have red supergiant progenitors. The SN ejecta after leaving the atmosphere of the star will presumably interact with dense $\left(\mathrm{n} \sim 10^{7}\right)$ 
circumstellar/interstellar medium. This interaction will produce a hot and luminous remnant with a life time of about 2 years. The predicted velocities, dimensions, temperature, density and luminosity of these remnants are similar to those of the canonical BLR. The emitted spectrum of a fast shock $\left(V=1000 \mathrm{~km} \mathrm{~s}^{-1}\right)$ in a high density $\left(n=10^{7}\right)$ environment has been studied by Daltabuit et al. (1978). They found that the emitted spectrum of the post shock cool dense shell, photoionized by the radiation of the shock, resembled that of a QSO. Thus in all aspects, we expect this rapidly evolving remnants to closely resemble the BLR.

Terlevich and Melnick (1988) provided evidence that this may be the case. They showed that the reported flare in the Seyfert type 1 NGC 5548, may have been the first detection of a SN in the nucleus of a galaxy, rather than an accretion event (Peterson and Ferland 1986). The spectrum of the flare looked very similar to that of SN $1983 \mathrm{~K}$ the only supernova known with a probable Wolf-Rayet progenitor (Niemela et al. 1985). Also the luminosity and duration of the flare were similar to those of SN 1983K.

Perhaps the most compelling evidence comes from some recent observations of supernovae. Filippenko (1989) has reported the discovery of a supernova in an HII region in one of the spiral arms of the SBc galaxy NGC 4615. After maximum the spectrum was dominated by broad permitted emission lines of hydrogen, FeII and CaII; it has a striking resemblance to the spectrum of a Seyfert type 1 or a QSO. A second supernova reported in the same paper, SN 1988I, showed a similar spectrum. Even the luminosities of the SNe were comparable to those of Seyfert 1 nuclei.

The previous discussion supports the idea that at least some low luminosity AGNs can be associated with nuclear star formation. To extend the model in order to include the high luminosity AGNs, it is necessary to discuss two fundamental observational constraints:

1 - the amplitude of the optical variability in QSOs, and

2 - the required star formation rate to explain the luminosity of the brightest QSOs.

III. Optical variability in QSOs. Long term monitoring of radio-selected quasars has shown that many are variable on time scales of years and with variable amplitudes (Tritton and Selmes 1971, Barbieri and Romano 1981, Pica and Smith 1983; PS83), and some may not vary at all. A small and perhaps unrepresentative subset, the Optically Violently Variable (OVV) are the most variable ones. Work on optically selected QSOs, has indicated that most of them show only small amplitude ( 0.2mag) variability (Bonoli et al. 1979, PS83). Perhaps the largest dataset is that obtained at the Rosemary Hill Observatory (PS83). It includes 13 years of continuous monitoring of $130 \mathrm{AGNs}$ and OVVs representing an average of 45 epochs per source. The average rms error of the photometry is 0.13 mag. PS 83 found a trend with luminosity in the variability amplitude. This is illustrated in Figure 1 were I plotted the observed variability amplitude versus the absolute blue luminosity estimated for $\mathrm{H}_{o}=50 \mathrm{~km} \mathrm{~s}^{-1} \mathrm{Mpc}^{-1}$ and $\mathrm{q}_{0}=0$. As can be seen from the figure luminous QSOs are somehow less variable than low luminosity QSOs and Seyfert nuclei. From least-squares fit to the data, 
PS83 estimated the change in peak-to-peak amplitude with luminosity as $0.12 \mathrm{mag} \pm 0.02 \mathrm{mag}$ per magnitude for all sources and $0.18 \pm 0.03 \mathrm{mag}$ per magnitude for the radio quasars only. This trend is expected if the variability is produced by random flares, but Pica and Smith did not favour this explanation because the slope of the observed relation is much more shallow than the theoretically expected. The variability amplitude should decrease roughly as the inverse of the square root of the luminosity. In their simple analysis Pica and Smith did not include the observational errors; this would tend to flatten the relation.

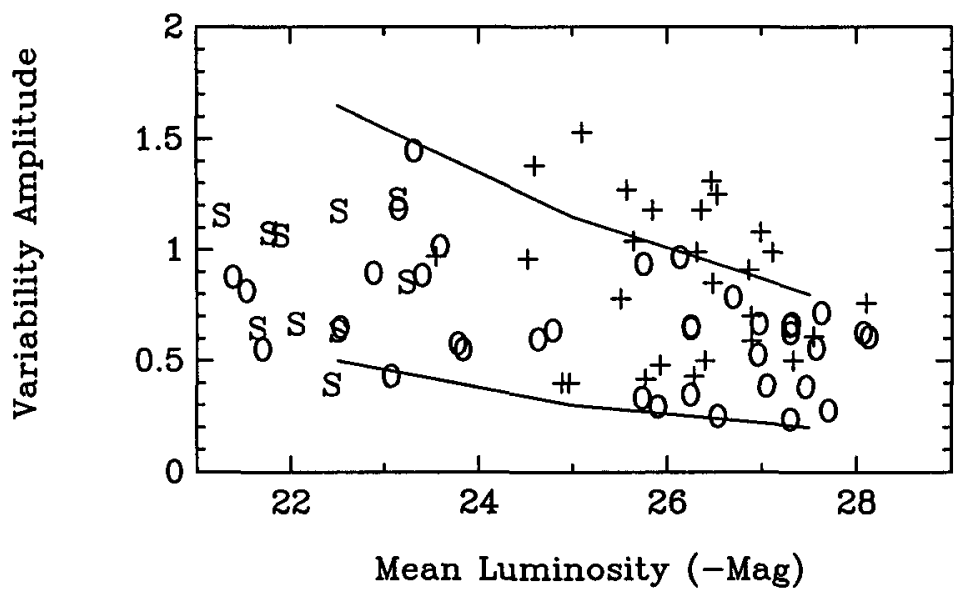

Figure 1 - Mean blue luminosity $\left(H_{o}=50, q_{o}=0\right)$ versus peak-to-peak variability amplitude for all non-OVV sources from the survey by PS83. $S=$ Seyfert galaxies; $O=$ optically selected QSOs; + = radio loud Quasars. The lines represent the expected range of values for a starburst.

To check this effect, and in collaboration with Alejandro Terlevich, I estimated using Montecarlo techniques, the variability of a Starburst during the supernova stage. We used the predicted light curve for a supernova and its remnant evolving in a $n=10^{7}$ density medium (Terlevich 1989). The light curve consists of the SN event lasting two months and reaching $\mathrm{M}_{b}=-19$. The $\mathrm{SN}$ remnant reaches a similar maximum luminosity 240 days after that initial explosion and then decays as $(t / 240 \text { days })^{-11 / 7}$. We constructed the light curve by superposition of random events with random amplitude between $M_{b}=-18$ and $M_{b}=-20$. Gaussian noise with an rms equal to the estimated observational errors by PS83 ( $\sigma=0.13 \mathrm{mg})$, was then added to this light curve. The resulting curve was then randomly sampled 45 times to reproduce the average number of epochs in the data set. With each light curve the sampling was repeated 100 times to estimate the rms change in the determination of the peak-to-peak amplitude. The lines in Figure 1 represent the result of the simulations. They correspond to the average variability amplitude plus and minus $2 \sigma$ fluctuations. They represent the range of values that we expect the observer to measure $95 \%$ of the time. We can see that most of the QSOs lie inside the predicted region. Only some radio selected quasars have variability slightly over the predicted values. 
We are lead to conclude that the variability observed in optically selected QSOs and in many radio selected quasars is well inside the expected in the starburst scenario. Therefore, the variability time scale is not constraining the size of the BLR; in our scenario the BLR of a luminous QSO, can be and probably is distributed over few $\mathrm{kpc}$ and still will produce the required variability.

If monitoring is performed with accuracy of 0.01 magnitudes then we predict variability amplitudes of

$\begin{array}{ll}0.10 \text { magnitudes for a } & M_{b}=-27.5 \text { QSO } \\ 0.30 \text { magnitudes for } a & M_{b}=-25.0 \text { QSO } \\ 0.85 \text { magnitudes for } a & M_{b}=-22.5 \text { QSO }\end{array}$

IV. Supernova rates in young galaxies and starbursts. A typical L* elliptical galaxy has a mass of about $10^{12} \mathrm{M}_{\odot}$ and an effective radius (half mass radius) of $5 \mathrm{kpc}$ and its present absolute blue luminosity is $M_{b}=-22$. The supernova rate (SNR) depends on the star-formation rate (SFR), and the details of the initial mass function (IMF). In the numerical examples that follow I assumed a Salpeter IMF. For this type of IMF one supernova explodes every 30-50 $\mathrm{M}_{\odot}$ of new stars, depending on the adopted lower and upper mass limits. Following Larson's dissipative models for the formation of elliptical galaxies, during the formation of the inner kiloparsec at the end of the collapse, the SFR for a $10^{12}$ $\mathrm{M}_{\odot}$ elliptical reaches a peak of about $3000 \mathrm{M}_{\odot} /$ year corresponding to about $100 \mathrm{SN} / \mathrm{year}$. If the blue luminosity of the $S N$ and $S N$ remnant is $M_{b} \sim 19.5$, and the remnants last for about 1 year, it is simple to estimate that the integrated blue luminosity of such a galaxy during the SN phase is about -25 . A very massive elliptical has a total mass of $10^{13} \mathrm{M}_{\odot}$ and during the formation of the inner part will reach SN rates of about $1000 \mathrm{SN} /$ year and $\mathrm{M}_{b}$ of -27 or -28 .

A different approach is to estimate the supernova rate for a burst of star formation that represents $5 \%$ of the total mass of the galaxy. Norman and Scoville (1988) have recently estimated the flux of stars off the main sequence for a Salpeter IMF cluster of stars following stellar evolution models by Renzini and Buzzoni (1986) and Iben and Renzini (1986). They find that the flux of stars off the main sequence is between 20 and 10 stars/year for a cluster of $10^{10} \mathrm{M}_{\odot}$ and for stellar masses larger than $7 \mathrm{M}_{\odot}$, i.e. inside the lower limit for type II SNe. The SN rate is almost equal to the flux of stars off the main sequence, therefore, a burst of strength $5 \%$ in a $10^{12} \mathrm{M}_{\odot}$ galaxy will also produce about $100 \mathrm{SN} / \mathrm{yr}$. This type of burst may be triggered by dynamical interactions, and presumably they are more common at high redshifts when galaxies had a larger gas content.

V. The size of the BLR. The size of the BLR will depend on the SN rate as a function of radius in a young elliptical galaxy. In Larson's model, the $\mathrm{SN}$ rate is at the maximum value at the end of the collapse and inside the effective radius. Assuming that the SN distribution 
follows the mass ditribution of the galaxy, we can then estimate its half intensity diameter or $2 \mathrm{xR}_{c}$. From the comparison of the $\mathbf{r}^{1 / 4}$ law and the Hubble law of brightness distribution Kerr (1957) found that $R_{h}=R_{e} / 11$ and as the $R_{c}$ in Hubble's law is $0.4142 \times R_{h}$ then in a typical elliptical $2 \mathrm{xR}_{c} \sim \mathrm{R}_{e} / 13$. In our typical $\mathrm{L}^{*}$ elliptical this corresponds to less than $400 \mathrm{pc}$ or 0.014 arcsec at a redshift of 1 .

A different estimate can be done using the scaling laws of bursts of star formation. Terlevich and Melnick (1981) and Melnick et al. (1987) studied the scaling laws for giant HII regions and HII galaxies. They found relations of the form $L \alpha \mathrm{R}^{2}$ are valid over more than an order of magnitude in radius. Applying the scaling to the determination of the core radius of 30-Doradus by Moffat et al. (1985) $(0.26 \mathrm{pc})$, and using a $\mathrm{M}_{b}=-14.5$, the predicted $\mathrm{R}_{c}$ for the $\mathrm{M}_{b}=-25$ burst of the $\mathrm{L}^{*}$ elliptical is less than $100 \mathrm{pc}$. I have to mention that the scaling law used implies constant surface density and the volume density is therefore smaller in the largest systems.

Putting both estimates together the size of the BLR in the starburts scenario should be,

$$
0.10 \operatorname{arcsec} 10^{-0.2 *\left(m_{b}-14.5\right)} \leq F W H M \leq 0.40 \operatorname{arcsec} 10^{-0.2 *\left(m_{b}-14.5\right)}
$$

where $\mathrm{m}_{b}$ is the apparent blue magnitude. The expression is valid for objects more luminous than $\mathrm{M}_{b}=-21$. The limit is to ensure that the BLR is not dominated by a single SN remnant.

VI. Concluding remarks. The ideas presented here demonstrate that the starburst model based in extremely simple assumptions is potentially able to reproduce the main observed properties of the BLR in Seyfert galaxies and QSOs.

The fact that the luminosities and variability of QSOs can be explained with the expected SN rates during the formation of a spheroidal galaxy leads naturally to the suggestion that perhaps most of the optically selected QSOs, representing the majority of the high redshift luminous objects, are young galaxies in the process of formation.

One important difference between the starburst and the blackhole scenario is the size of the BLR. The fact that the BLR should be tens of parsecs FWHM in luminous Seyferts, constitutes a potential test for the scenario. Based in simple scaling laws I predict that the HST may be able to resolve the BLR of some of the nearest luminous Seyfert galaxies.

A second test is based in the predicted relation between variability amplitude and luminosity. This second test requires $0.01 \mathrm{mag}$ photometry of few luminous QSOs during 3 or 4 years.

I would like to thank Elena Terlevich for her comments that greatly improved this paper. 


\section{References}

Adams, T.F. and Weedman, D.W., 1975. Astrophys. J., 199, 19.

Baldwin, J.A., Phillips, M.M. and Terlevich, R., 1981. Publ. astr. Soc. Pacif, , 93, 5.

Barbieri, C. and Romano, G., 1981. Astr. Astrophys. Suppl. , 44, 159.

Barlow, M. and Cohen, M., 1982. in: Wolf-Rayet Stars: Observations, Physics, Evolution, p. 387, eds de Loore,C. and Willis, A. Reidel, Dordrecht, Holland.

Bonoli, F., Braccesi, A., Federici, L., Zitelli, V. and Formiggini, L., 1979. Astr. Astrophys. Suppl. , $35,391$.

Chiosi, C., 1981. in: The most massive stars, p.261, eds D'Odorico, S.Baade and Kjar,K. (Proceedings ESO workshop)

Conti, P.S., 1976. Mem.Soc.R.Sci.Liege, 6eme Serie 9, 193.

Cowley, A.P., Crampton, D. and McClure, R.D., 1982. Astrophys. J. , 263, 1.

Daltabuit, E., MacAlpine, G. and Cox, D., 1978. Astrophys. J. , $219,372$.

Davidson, K. and Kinman, T.D., 1982. Publ. astr. Soc. Pacif., 94, 634.

Field, G.B., 1964. Astrophys. J. , 140, 1434.

Filippenko, A.V., 1989. Astron. J. , 97, 726.

Harwit, M. and Pacini, F., 1975. Astrophys. J. Lett., 200, 1127.

Heckmann, T.M., Balick, B. and Crane, P.C., 1980. Astr. Astrophys. Suppl. , 40, 295.

Iben, I. and Renzini, A., 1986. Ann. Rev. Astr. Astrophys., 21, 271.

Kerr, F.J., 1957. Astron. J. , 62, 93.

Larson, R.B., 1974. Mon. Not. R. astr. Soc. , 166, 585.

Maeder, A., 1983. Astr. Astrophys., 120, 113.

McCrea, W.H., 1976. in: The galaxy and the local group, eds Dickens, R.J. and Perry, J.E. (RGO Bull 182).

Melnick, J., Moles, M.,Terlevich, R. and Garcia-Pelayo, J-M, 1987. Mon. Not. R. astr. Soc., 226, 849.

Moftat, J.W., Seggewiss, W. and Shara, M.M., 1985. Astrophys. J. , 295, 109.

Norman, C. and Scoville, N., 1988. Astrophys. J., 332, 124

Osterbrock, D.E., 1978. Phys. Scripta, 17, 285.

Perry, J.J. and Dyson, J.E., 1985. Mon. Not. R. astr. Soc. , 213, 665.

Peterson, B.M. and Ferland, G., 1986. Nature, 324, 345.

Pica, A.J. and Smith, A.G., 1983. Astrophys. J. , 272, 11; (PS83).

Pronik, I.I., 1974. Soviet astr., 16, 628.

Rees, M.J., 1978. Ann. N.Y. Acad. Sci., 320, 613.

Rees, M.J., 1984. Ann. Rev. Astr. Astrophys. , 22, 471.

Renzini, A. and Buzzoni, A., 1986. in: Spectral evolution in Galaxies, eds. C.Chiosi and A. Renzini, p. 195, Reidel.

Shklovskii, I.S., 1960. Soviet astr., 4, 885.

Tanaka, S., 1966. Publ. astr. Soc. Japan, 18, 47.

Terlevich, R., 1988. in The post recombination Universe, eds. N. Kaiser and A.N. Lasenby, Kluwer.

Terlevich, R., 1989, in Evolutionary phenomena in Galaxies, eds. J.E. Beckman and B.E.J. Pagel, Cambridge Univ. Press.

Terlevich, R., Melnick, J. and Moles, M., 1987, in Observational evidence for activity in galaxies, eds. E.Ye. Khachikyan, K.J. Fricke and J. Melnick, Reidel, Dordrecht; (TMM87).

Terlevich, R and Melnick J., 1987. in Starburst and galaxy evolution eds. T.X. Thuan, T. Montmerle and J. Tran Than Van, Frontiere.

Terlevich, R. and Melnick, J., 1988. Nature, 333, 239.

Terlevich, R. and Melnick, J., 1981. Mon. Not. R. astr. Soc. , 195, 839.

Terlevich, R. and Melnick, J., 1985. Mon. Not. R. astr. Soc., 213, 841; (TM85).

Terlevich, R. and Terlevich, A., 1990. in preparation.

Tritton, K.P. and Selmes, R.A., 1971. Mon. Not. R. astr. Soc. , 153, 453.

Weedman, D.W., 1983. Astrophys. J. , 266, 479. 


\section{Discussion}

PAGEL: What SN rate do you need to account for the variability of the most luminous AGNs?

TERLEVICH: Three SN a day keep the blackholes away.

OSTERBROCK: It is very important to explore all possible models for interpreting AGNs, such as this one which is quite different from the more conventional blackhole picture. The basic feature of AGN spectra is photoionization by a spectrum that extends to high energies; clearly if very high temperature stars actually exist, assembling enough of them will give almost any desired photoionizing spectrum. Detailed predictions of the profiles and variations should help to distinguish between proposed models and are clearly important. The difficulty I had with such proposals is that the best models I know, and the observational data I know, do not suggest that Wolf-Rayet stars emit high-energy photoionizing spectra.

TERLEVICH: Wolf-Rayet stars cover a large range of temperatures, most of them are relatively cool stars, but for a minority mainly composed of extreme WC and WO stars the evidence suggests very high effective temperature. Probably the best case is reported by Davidson and Kinman (1982). They obtained observations of an HII region ionized by a WC5 or WC4. From the analysis of the emission lines they comcluded that the ionizing radiation peaked at $37 \mathrm{ev}$ similar to the peak of a $110,000 \mathrm{~K}$ blackbody. The luminosity of this WC star is $1.3 \times 10^{5} \mathrm{~L}_{\odot}$.

WAMPLER: You have presented an interesting alternative to the 'standard' model. The standard model is not troubled by observations of high polarization in the continuum. I wonder if detailed polarization measurements might be able to distinguish your 'thermal' model from the standard non-thermal model.

TERLEVICH: I believe it can give some discrimination between thermal vs. non-thermal models specially if variable polarization is detected. My difficulty is that I do not believe that the canonical accretion disk + blackhole model is fundamentally non-thermal. I'm shure it may be possible to create scenarios in which most of the luminosity is emitted by thermal processes.

LARSON: I would worry about the stupendously high star formation rates required in your picture. You require 1,000 SNe per year, which in turn require a star formation rate of order $100,000 \mathrm{M}_{\odot}$ per year. Even allowing $10^{10} \mathrm{M}_{\odot}$ of material to participate in this phenomenon, it can only last for $10^{5}$ years, which makes it a very short-lived and therefore very rare phenomenon. 
TERLEVICH: You have picked the most extreme case. It corresponds to the most luminous QSOs. For this case I used a very massive elliptical galaxy with total mass $10^{13} M_{\odot}$ and your models for galaxy formation. The peak star formation rate is, depending on the model, between $50,000 \mathrm{M}_{\odot} / \mathrm{yr}$ and $20,000 \mathrm{M}_{\odot} / \mathrm{yr}$ for about $10^{8}$ years. This gives a SN rate between $2,000 \mathrm{SN} /$ year and $200 \mathrm{SN} /$ year depending on the choice of IMF parameters. This rate and life-time are exactly what is needed to scale the space density of present day luminous elliptical galaxies to the space density in co-moving coordinates of redshift 2 luminous QSOs.

DYSON: Have you taken into account the fact that the SN remnants are evolving in a very high, presumably non-thermal radiation field, which would affect the physics via, for example, Compton cooling?

TERLEVICH: The radiation field is not expected to be intense. These SN remnants are not concentrated in a small volume around the active nucleus like in your scenario. They are spread over kiloparsecs, they can even cover most of the galaxy. Compton cooling should be effective only in the early stages of the evolution of the remnant.

DULTZIN-HAYAN: I believe that you are leaving out of the scenario not only OVV and BL Lacs but also radio galaxies. How can you possible collimate radio-jets in an 'only SN' scenario?

TERLEVICH: I agree, it is very difficult to explain the collimation of objects like Cygnus A. On the other hand most objects classified as AGNs either do not have radio emission or if they do, the ejecta is not highly collimated. 